\title{
SOLUCION GLOBAL Y DECAIMIENTO DE LA ENERGIA DE SOLUCIONES PARA UNA CLASE DE ECUACIONES ABSTRACTAS ASOCIADAS A LA ECUACION NO-LINEAL DE LA VIGA CON TERMINO DISIPATIVO
}

\author{
Raúl Izaguirre Maguiña \\ Eugenio Cabanillas Lapa
}

RESUMEN.- En este trabajo estudiamos la existencia, unicidad y el decaimiento de la energía asociada a la solución débil para el problema abstracto:

$$
\text { * }^{*} \quad \begin{cases}u^{\prime \prime}+M\left(\left|A^{1 / 2} u\right|^{2}\right) A u+B u^{\prime}=f \\ u(0)=u_{0} ; & ; \quad u^{\prime}(0)=u_{1} .\end{cases}
$$

El operador lineal $A$, esta definido por la terna $\{H, V,(())$,$\} , donde H$ y $V$, son espacios de Hilbert, la inmersión de Ven Hes densa y compacta. La función no - lineal $M(s)$ es una función real derivable, estrictamente positiva, $B: H \rightarrow H$ es un operador lineal positivo.

\section{INTRODUCCIÓN}

Sea $\Omega$ un abierto de $\mathrm{R}^{n}$ con frontera regular; $Q$ el cilindro $\Omega \times] 0, T[, 0<T<\infty$, con frontera lateral $\Sigma$. La siguiente ecuación diferencial parcial no-lineal

(1)

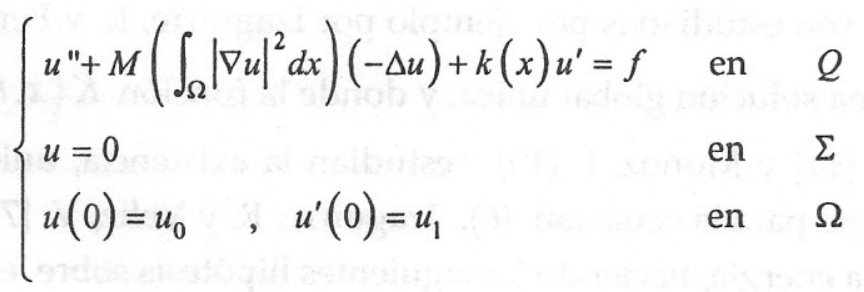

es un modelo n-dimensional con término disipativo lineal, de la ecuación que describe las vibraciones no-lineales de una cuerda elástica de extensión finita, que para dimensión $n=1$, tiene la forma (ver [17])

$$
\frac{\partial^{2} u}{\partial t^{2}}-\left(\frac{H}{\xi}+\frac{E A}{2 \xi L} \int_{0}^{L}\left(\frac{\partial u}{\partial x}\right)^{2} d x\right) \frac{\partial^{2} u}{\partial x^{2}}+k(x) \frac{\partial u}{\partial t}=0
$$


donde $E$ es el módulo de Young del material, I el momento de inercia, $\xi$ y $A$ representan la densidad y el área de la sección recta, respectivamente.

Asimismo, desde el punto de vista de los métodos abstractos en espacios de Hilbert, la ecuación (1) puede ser considerada como un caso particular de

$$
\left\{\begin{array}{l}
u^{\prime \prime}(t)+M\left(\left|A^{1 / 2} u(t)\right|^{2}\right) A u(t)+B u^{\prime}(t)=f(t) \quad \text { en } \quad H \\
u(0)=u_{0} \quad, \quad u^{\prime}(0)=u_{1}
\end{array}\right.
$$

donde $A$ es un operador autoadjunto, no acotado y positivo, definido por la terna $\{H, V,(())\}, H,$,$V son espacios de Hilbert, la inmersión de V$ en $H$ es densa y compacta y $B$ es un operador simétrico y coercitivo.

Variantes de la ecuación abstracta (3), son por ejemplo:

$$
\begin{aligned}
& u^{\prime \prime}+M\left(\left|A^{1 / 2} u\right|^{2}\right) A u+A^{\alpha} u^{\prime}=f \\
& u^{\prime \prime}+M\left(\left|A^{1 / 2} u\right|^{2}\right) A u+\left|u^{\prime}\right|^{\rho} u^{\prime}=f \\
& K u^{\prime \prime}+A^{2} u+M\left(\left|A^{1 / 2} u\right|^{2}\right) A u+u^{\prime}=f .
\end{aligned}
$$

La ecuación (4), es estudiada entre otros por Medeiros, L. A. y Milla, M. M. [12], quienes prueban la existencia de soluciones globales para $M(s) \geq m_{0}>0$, $A$ un operador estrictamente positivo y $\alpha \in(0,1]$. Soluciones globales para ecuaciones del tipo (6), son estudiadas por ejemplo por Izaguirre, R. y Pereira, D. [6], quienes obtienen una solución global única, y donde la función $K(x, t)$ es no negativo. Pereira, D. [14] y Muñoz, J. [13] estudian la existencia, unicidad y decaimiento de la energía para la ecuación (6). Izaguirre, R. y Veliz, V. [7] obtienen el decaimiento de la energía, haciendo las siguientes hipótesis sobre el operador acotado $K$ y la función no-lineal $M$ :

(i) $M(s) \geq-\sigma, \forall s \geq 0$, y $0<\sigma<\lambda_{1} ; \lambda_{1}$ es el primer auto valor de $A$.

(ii) $K$ es un operador simétrico en $H$, tal que $(K u, u) \geq 0 ; \forall u \in H$.

En la referencia [2] A'assila demuestra la existencia , unicidad y decaimiento de la energía para soluciones de los tipos anteriores.

Soluciones globales con datos analíticos "suficientemente pequeños" son estudiadas en [8]. 
El caso degenerado de la ecuación (6) es tratado también en la referencia [7], donde se determina la existencia y unicidad de una solución local débil. Asimismo en Izaguirre, R. y Cabanillas, E. [6'] se trata la ecuación abstracta

(8)

$$
\left\{\begin{array}{l}
u^{\prime \prime}+A^{\varepsilon} u+M\left(\left|A^{\alpha} u\right|^{2}\right) A^{\beta} u+u^{\prime}=f \quad \text { en } H \\
u(0)=u_{0} \quad, \quad u^{\prime}(0)=u_{1}
\end{array}\right.
$$

donde la función no lineal $M$ es de clase $C^{1}$ y acotada inferiomente; el operador $A$ es no negativo y los exponentes no negativos $\alpha, \beta, \varepsilon$; verifican ciertas condiciones técnicas.

\section{PRELIMINARES}

Sean $(V,(())),,(H,()$,$) espacios de Hilbert, la inmersión de V$ en $H$ densa y compacta. Sea también $A$ el operador definido por la terna $\{V, H,(())$,$\} .$

Entonces, $D(A)$ es un subespacio denso en $H$; $A$ es un operador no acotado, auto adjunto y positivo de $H$ con espectro discreto

$$
A w_{v}=\lambda_{\nu} w_{\nu}, \quad \forall v=1,2, \ldots ; \lambda_{v} \rightarrow \infty \text { cuando } v \rightarrow \infty
$$

donde $\left\{w_{\nu}\right\}$ es un sistema ortonormal completo de $H$ de modo que :

$$
\begin{aligned}
& D(A)=\left\{u \in H ; \sum_{v=1}^{\infty} \lambda_{v}^{2}\left|\left(u, w_{v}\right)\right|^{2}<\infty\right\} \\
& A u=\sum_{v=1}^{\infty} \lambda_{v}^{2}\left(u, w_{v}\right) w_{v} \quad u \in D(A) .
\end{aligned}
$$

Asimismo, el operador $A^{1 / 2}$ esta bien definido; es decir

$$
\begin{aligned}
& D\left(A^{1 / 2}\right)=\left\{u \in H ; \sum_{v=1}^{\infty} \lambda_{v}\left|\left(u, w_{v}\right)\right|^{2}<\infty\right\}=V \\
& A^{1 / 2} u=\sum_{v=1}^{\infty} \lambda_{v}^{1 / 2}\left(u, w_{v}\right) w_{v} \quad u \in V .
\end{aligned}
$$


Definiendo

$$
|u|_{\alpha}^{2}=\sum_{v=1}^{\infty} \lambda_{v}^{2 \alpha}\left|\left(u, w_{v}\right)\right|^{2}, u \in D\left(A^{\alpha}\right)
$$

se tiene que:

1) $\left(D\left(A^{\alpha}\right),|\cdot| \alpha\right)$ es un espacio de Hilbert.

2) Si $\alpha>\beta, D\left(A^{\alpha}\right) \subset D\left(A^{\beta}\right)$ y la inmersión de $D\left(A^{\alpha}\right)$ en $\subset D\left(A^{\beta}\right)$ es compacta.

Bajo estas consideraciones teóricas de los métodos en espacios de Hilbert para tratar ecuaciones diferenciales parciales, establecemos el primer teorema sobre unicidad de la solución para el problema planteado.

La funcion (no lineal) $M$ satisface las siguientes condiciones :

$\mathrm{H}-1: M \in C^{1}(\mathbf{R})$

$\mathrm{H}-2: M(s) \geq m_{0}>0 ; \quad \forall s \in \mathbf{R}$

H-3 : $\hat{M}(s)=\int_{0}^{s} M(\lambda) d \lambda \leq m_{1} s M(s)$

H-4: El operador lineal lineal $B: H \rightarrow H$ es simétrico y coercitivo :

$$
\begin{aligned}
& k_{0}|u|^{2} \leq(\mathrm{B} u, u) \\
& |(B u, v)| \leq k_{1}|u||v|
\end{aligned}
$$

H-5 : Existen operadores lineales simétricos $A_{i}: V \rightarrow H, B_{i} H \rightarrow H$; tales que:

$$
\begin{aligned}
& b(u, v)=(A u, K v)=\sum_{i=1}^{n}\left(K A_{i} u, A_{i} v\right)+\sum_{i=1}^{n}\left(K_{i} u, A_{i} v\right) . \\
& a_{1}\left|A^{1 / 2} u\right|^{2} \leq \sum_{i=1}^{n}\left|A_{1} u\right|^{2} \leq a_{2}\left|A_{i} u\right|^{2}
\end{aligned}
$$

Observacion 1. De la condición H-5 obtenemos:

$$
\begin{aligned}
& b(u, u) \geq k_{0} \quad a_{1}\left|A_{i} u\right|^{2}+\sum_{i=1}^{n}\left(K_{i} u, A_{i} v\right) \\
& \left|\sum_{i=1}^{n}\left(K_{i} u, A_{i} v\right)\right| \leq a_{3}|u|\left|A^{1 / 2} v\right| .
\end{aligned}
$$


TEOREMA DE EXISTENCIA GLOBAL. Sean $u_{0} \in D(A), u_{1} \in D\left(A^{1 / 2}\right)$ tales que, $\left|A u_{0}\right|^{2}+\left|A^{1 / 2} u_{1}\right|^{2} \leq R^{2}$; donde la constante $R$ será determinada posteriormente. Supongamos que la función $M y$ el operador $B$ satisfacen las condiciones $H-1, \ldots, H-5$, entonces existe una función vectorial $u:[0, T] \rightarrow D(A)$, tal que :

$$
\begin{aligned}
& u \in L^{\infty}(0, T ; D(A)) \\
& u^{\prime} \in L^{\infty}\left(0, T ; D\left(A^{1 / 2}\right)\right) \\
& u^{\prime \prime} \in L^{2}(0, T ; H) \\
& u^{\prime \prime}+M\left(\left|A^{1 / 2} u\right|^{2}\right) A u+B u^{\prime}=f \text { en } L^{2}(0, T ; H) \\
& u(0)=u_{0}, u^{\prime}(0)=u_{1} .
\end{aligned}
$$

Demostracion . Sean $V_{m}=\left[w_{1}, \ldots, w_{m}\right]$, el subespacio generado por los primeros " $m$ " vectores propios del operador $A$ y

$$
u_{m}(t)=\sum_{i=1}^{m} g_{i m}(t) w_{i} \in V_{m}
$$

Donde las funciones $g_{\text {im }}$, son determinadas por la solución del siguiente sistema de ecuaciones diferenciales ordinarias no lineales :

$$
\begin{aligned}
& \left(u_{m}^{\prime \prime}(t), w_{j}\right)+M\left(\left|A^{1 / 2} u_{m}(t)\right|^{2}\right)\left(A u_{m}(t), w_{j}\right)+\left(B u_{m}^{\prime}(t), w_{j}\right)=0, \quad j=1,2, \ldots, m \\
& \text { (12) } u_{m}(0)=u_{0 m} \rightarrow u_{o} \quad \text { en } \quad D(A) \\
& \text { (13) } u_{m}^{\prime}(0)=u_{1 m} \rightarrow u_{1} \quad \text { en } \quad D\left(A^{1 / 2}\right)
\end{aligned}
$$

Luego de un análisis y aplicación del Teorema de Caratheodory sobre existencia local de solución de ecuaciones diferenciales ordinarias no lineales, el sistema anterior admite solución en un intervalo $\left[0, t_{m}\right)$, de donde se sigue la existencia de las soluciones aproximadas $u_{m}$ para $m \geq 1$. A continuación, debemos obtener estimados apriori (acotaciones), para la sucesión $\left\{u_{m}\right\}$, de modo que, podamos prolongarlas uniformemente a un intervalo de existencia.

ESTIMADO APRIORI 1. Es fácil comprobar que la ecuación (11) se verifica, reemplazando $w_{i}$ por $v \in V_{m}$. Entonces, haciendo $v=2 u_{m}^{\prime}(t) \in V_{m}$, obtenemos:

$$
\frac{d}{d t}\left\{\left|u_{m}^{\prime}(t)\right|^{2}+\hat{M}\left(\left|A^{1 / 2} u_{m}(t)\right|^{2}\right)\right\}+2\left|B^{1 / 2} u_{m}^{\prime}(t)\right|^{2}=0
$$


Entonces integrando en la desigualdad (14), sobre $[0, t)$ se obtiene

$$
\begin{aligned}
& \left|u_{m}^{\prime}(t)\right|^{2}+\hat{M}\left(\left|A^{1 / 2} u_{m}(t)\right|^{2}\right)+2 \int_{0}^{t}\left|B^{1 / 2} u_{m}^{\prime}(s)\right|^{2} d s \leq \\
& \quad \leq\left|u_{1 m}\right|^{2}+\hat{M}\left(\left|A^{1 / 2} u_{0 m}\right|^{2}\right) \leq\left|u_{1}\right|^{2}+\hat{M}\left(\left|A^{1 / 2} u_{0}\right|^{2}\right) .
\end{aligned}
$$

ESTIMADO APRIORI 2.- Haciendo $v=2 A u_{m}^{\prime}(t)$ en la ecuación aproximada obtenemos,

$$
\begin{array}{r}
\frac{d}{d t}\left\{\left|A^{1 / 2} u_{m}(t)\right|^{2}+\psi_{m}(t)\left|A^{1 / 2} u_{m}(t)\right|^{2}\right\}=-2 b\left(u_{m}^{\prime}(t), u_{m}^{\prime}(t)\right)+\psi_{m}^{\prime}(t)\left|A u_{m}(t)\right|^{2} \leq \\
\leq-2 a_{3}\left|A^{1 / 2} u_{m}^{\prime}(t)\right|^{2}+\sum_{i=1}^{n}\left(B_{i} u_{m}^{\prime}(t), A_{i} u_{m}^{\prime}(t)\right)+\psi_{m}^{\prime}(t)\left|A u_{m}(t)\right|^{2}
\end{array}
$$

donde

$$
\begin{aligned}
& \psi_{m}(t)=M\left(\left|A^{1 / 2} u_{m}(t)\right|^{2}\right) \\
& \psi_{m}^{\prime}(t)=2 M^{\prime}\left(\left|A^{1 / 2} u_{m}(t)\right|^{2}\right)\left(A^{1 / 2} u_{m}(t), A^{1 / 2} u_{m}^{\prime}(t)\right) .
\end{aligned}
$$

Ahora teniendo en cuenta el estimado (15) y la continuidad de la función $M$ obtenemos

$$
\begin{aligned}
\left|\psi_{m}^{\prime}(t)\right| & =\left|2 M^{\prime}\left(\left|A^{1 / 2} u_{m}(t)\right|^{2}\right)\left(A^{1 / 2} u_{m}(t), A^{1 / 2} u_{m}^{\prime}(t)\right)\right|= \\
& =\left|2 M^{\prime}\left(\left|A^{\alpha} u_{m}(t)\right|^{2}\right)\left(A u_{m}(t), u_{m}^{\prime}(t)\right)\right| \\
& \leq C\left|A u_{m}(t)\right|\left|u_{m}^{\prime}(t)\right| .
\end{aligned}
$$

Integrando en (16), utilizando la desigualdad de Cauchy - Schwarz , (19) , y la condición H-1, obtenemos

$$
\begin{aligned}
& \left|A^{1 / 2} u_{m}^{\prime}(t)\right|^{2}+\psi_{m}(t)\left|A^{1 / 2} u_{m}(t)\right|^{2} \leq\left|A^{1 / 2} u_{1 m}\right|^{2}+\psi_{m}(0)\left|A u_{0 m}\right|^{2}+ \\
& +C \int_{0}\left\{\left|A^{1 / 2} u_{m}^{\prime}(s)\right|^{2}+\left|A u_{m}(s t)\right|^{2}\right\} d s \leq C+C \int_{0}^{t}\left\{\left|A^{1 / 2} u_{m}^{\prime}(s)\right|^{2}+\left|A u_{m}(s t)\right|^{2}\right\} d s .
\end{aligned}
$$

Aplicando el lema de Gronwall en (20) obtenemos la acotación

$$
\eta(t)=\left|u_{m}^{\prime}(t)\right|^{2}+\left|A^{\varepsilon / 2} u_{m}(t)\right|^{2} \leq C \quad \forall t \in[0, T]
$$

ESTIMADO APRIORI 3. Haciendo $v=2 A u_{m}(t)$ en la ecuación aproximada obtenemos 
(22) $\begin{aligned} \frac{d}{d t} & \left\{\left(A^{1 / 2} u_{m}^{\prime}(t), A^{1 / 2} u_{m}(t)\right)+\frac{1}{2} \sum_{i=1}^{n}\left|B^{1 / 2} u_{m}(t)\right|^{2}\right\}=\left|A^{1 / 2} u_{m}^{\prime}(t)\right|^{2}-\psi_{m}(t)\left|A u_{m}(t)\right|^{2} \\ & +\sum_{i=1}^{n}\left(B_{i} u_{m}^{\prime}(t), A_{i} u_{m}^{\prime}(t)\right) \leq\left|A^{1 / 2} u_{m}^{\prime}(t)\right|^{2}-\psi_{m}(t)\left|A^{1 / 2} u_{m}(t)\right|^{2}+C_{4}\left|u_{m}^{\prime}(t)\right|\left|A u_{m}(t)\right|\end{aligned}$

donde

$$
C_{4}=a_{3} d_{1}
$$

Definimos :

$$
\begin{aligned}
& E_{0}(t)=\left|u_{m}^{\prime}(t)\right|^{2}+\hat{M}\left(\left|A^{1 / 2} u_{m}(t)\right|^{2}\right) \\
& E_{1}(t)=\left|A^{1 / 2} u_{m}^{\prime}(t)\right|^{2}+M\left(\left|A^{1 / 2} u_{m}(t)\right|^{2}\right)\left|A u_{m}(t)\right|^{2} \\
& H(t)=\left(A^{1 / 2} u_{m}^{\prime}(t), A^{1 / 2} u_{m}(t)\right)+\frac{1}{2} \sum_{i=1}^{n}\left|B^{1 / 2} A_{i} u_{m}(t)\right|^{2} .
\end{aligned}
$$

(Para simplificar las notaciones, en lo sucesivo, prescindiremos de los símbolos "m" y " $t$ ").

Por los estimados previamente obtenidos tenemos que :

$$
\begin{aligned}
& E_{o}^{\prime}=-2\left|B^{1 / 2} u^{\prime}\right|^{2} \\
& E_{1}^{\prime}=-2 b\left(u^{\prime}, u^{\prime}\right)+\psi^{\prime}(t)|A u|^{2} \\
& H^{\prime}=\left|A^{1 / 2} u^{\prime}\right|^{2}-\psi(t)|A u|^{2}-\sum_{i=1}^{n}\left(B_{i} u^{\prime}, A_{i} u\right) .
\end{aligned}
$$

Para $\alpha, \beta$ positivos, hacemos:

$$
S=E_{0}+\alpha E_{1}+\beta H
$$

Proposición 1. Existen constantes $\gamma_{0}, \gamma_{1}$ números reales positivos tales que:

$$
\gamma_{0} E_{1} \leq S \leq \gamma_{1} E_{1} \quad y \quad \gamma_{0}<\gamma_{1}
$$

Demostración. Tenemos que,

$$
\begin{aligned}
S=E_{0}+\alpha E_{1}+\beta H & =\left|u_{m}^{\prime}\right|^{2}+\hat{M}\left(\left|A^{1 / 2} u_{m}\right|^{2}\right)+\alpha\left|A^{1 / 2} u_{m}^{\prime}\right|^{2}+\alpha M\left(\left|A^{1 / 2} u_{m}\right|^{2}\right)\left|A u_{m}\right|^{2}+ \\
& +\beta\left(A^{1 / 2} u_{m}^{\prime}(t), A^{1 / 2} u_{m}\right)+\frac{\beta}{2} \sum_{i=1}^{n}\left|B^{1 / 2} A_{i} u_{m}\right|^{2}
\end{aligned}
$$


Por el estimado apriori 1 :

$$
\begin{aligned}
E_{0}=\left|u^{\prime}\right|^{2}+\hat{M}\left(\left|A^{1 / 2} u\right|^{2}\right) & \leq d_{1}\left|A^{1 / 2} u^{\prime}\right|^{2}+m_{1} M\left(\left|A^{1 / 2} u\right|^{2}\right)\left|A^{1 / 2} u\right|^{2} \\
& \leq d_{1}\left|A^{1 / 2} u^{\prime}\right|^{2}+m_{1} d_{1} M\left(\left|A^{1 / 2} u\right|^{2}\right)|A u|^{2} \leq C_{5} E_{1}
\end{aligned}
$$

donde

$C_{5}=d_{1} \operatorname{Max}\left\{1, m_{1}\right\}$. Además,

$$
\begin{aligned}
\beta H & =\beta\left(A^{1 / 2} u^{\prime}, A^{1 / 2} u\right)+\frac{\beta}{2} \sum_{i=1}^{n}\left|B^{1 / 2} A_{i} u\right|^{2} \leq \\
& \leq \frac{\beta}{2}\left|A^{1 / 2} u^{\prime}\right|^{2}+\frac{\beta d_{1}}{2}|A u|^{2}+\frac{\beta k_{0}}{2} \sum_{i=1}^{n}\left|A_{i} u\right|^{2} \leq \\
& \leq \frac{\beta}{2}\left|A^{1 / 2} u^{\prime}\right|^{2}+\frac{\beta d_{1}}{2 m_{0}} M\left(\left|A^{1 / 2} u\right|^{2}\right)|A u|^{2}+\frac{\beta d_{1} a a_{1} k_{0}}{2 m_{0}} M\left(\left|A^{1 / 2} u\right|^{2}\right)|A u|^{2} \leq \\
& \leq \frac{\beta}{2} \operatorname{Max}\left\{1, \frac{d_{1}}{m_{0}}, \frac{k_{0} a_{1} d_{1}}{m_{0}}\right\}\left\{\left|A^{1 / 2} u^{\prime}\right|^{2}+M\left(\left|A^{1 / 2} u\right|^{2}\right)|A u|^{2}\right\}=C_{6} E_{1}
\end{aligned}
$$

Luego :

$$
\begin{aligned}
& S \leq\left(C_{5}+C_{6}+\alpha\right) \quad E_{1} \leq \gamma_{1} \quad E_{1} \\
& \gamma_{1}=3 \operatorname{Max}\left\{C_{5}, C_{6}, \alpha\right\}
\end{aligned}
$$

Por otro lado,

$$
\begin{aligned}
\beta H & =\beta\left(u^{\prime}, A u\right)+\frac{\beta}{2} \sum_{i=1}^{n}\left|B^{1 / 2} A_{i} u\right|^{2} \geq \\
& \geq-\left|u^{\prime}\right|^{2}+\frac{\beta^{2}}{4}|A u|^{2}-\frac{\beta d_{1} a_{1} k_{1}}{2 m_{0}} M\left(\left|A^{1 / 2} u\right|^{2}\right)|A u|^{2} \leq \\
& \geq-\left|u^{\prime}\right|^{2}-\left(\frac{\beta^{2}}{4 m_{0}}+\frac{\beta d_{1} a_{1} k_{1}}{2 m_{0}}\right) M\left(\left|A^{1 / 2} u\right|^{2}\right)|A u|^{2} \geq \\
& \geq-E_{0}-\left(\frac{\beta^{2}}{4 m_{0}}+\frac{\beta d_{1} a_{1} k_{1}}{2 m_{0}}\right) M\left(\left|A^{1 / 2} u\right|^{2}\right)|A u|^{2}
\end{aligned}
$$

Haciendo $C(\beta)=\left(\frac{\beta^{2}}{4 m_{0}}+\frac{\beta d_{1} a_{1} k_{1}}{2 m_{0}}\right)$ y $\alpha=2 C(\beta)$, obtenemos

$$
S=E_{0}+2 C(\beta) E_{1}+\beta H \geq C(\beta) E_{1}, \quad \forall \beta>0
$$


Considerando $\beta$ suficientemente pequeño, $\gamma_{0}=C(\beta)<\gamma_{1}$.

Proposición 2. Existen constantes positivas $p, q$ tales que,

$$
S^{\prime}(t) \leq-p S(t)+q E_{1}^{1 / 2}(t) S(t)
$$

Demostración. Tenemos

$$
\begin{aligned}
& E_{o}^{\prime}=-2\left|B^{1 / 2} u^{\prime}\right|^{2} \leq-2 k_{0}\left|u^{\prime}\right|^{2} \\
& 2 C(\beta) E_{1}^{\prime}=-4 C(\beta) b\left(u^{\prime}, u^{\prime}\right)+2 C(\beta) M^{\prime}\left(\left|A^{1 / 2} u\right|\right)\left(A^{1 / 2} u, A^{1 / 2} u^{\prime}\right)|A u|^{2} \\
& \beta H^{\prime}=\beta\left|A^{1 / 2} u^{\prime}\right|^{2}-\beta M\left(\left|A^{1 / 2} u\right|\right)|A u|^{2}-\beta \sum_{i=1}^{n}\left(B_{i} u^{\prime}, A_{i} u\right)
\end{aligned}
$$

Entonces

$$
\begin{aligned}
S^{\prime} & \leq-2 k_{0}\left|u^{\prime}\right|^{2}-4 C(\beta) k_{0} a_{0}\left|A^{1 / 2} u^{\prime}\right|^{2}\left(u^{\prime}, u^{\prime}\right)+4 C(\beta) a_{3}\left|u^{\prime}\right|\left|A^{1 / 2} u^{\prime}\right|+ \\
& +4 C(\beta) a_{5}\left|A^{1 / 2} u^{\prime}\right||A u|+\beta\left|A^{1 / 2} u^{\prime}\right|^{2}-\beta M\left(\left|A^{1 / 2} u\right|\right)|A u|^{2}+\beta a_{3}\left|u^{\prime}\right|\left|A^{1 / 2} u\right| \leq \\
& \leq-2 k_{0}\left|u^{\prime}\right|^{2}-4 C(\beta) k_{0} a_{0}\left|A^{1 / 2} u^{\prime}\right|^{2}\left(u^{\prime}, u^{\prime}\right)+2 C(\beta) a_{3}\left(\varepsilon_{1}\left|u^{\prime}\right|^{2}+\frac{1}{\varepsilon_{1}}\left|A^{1 / 2} u^{\prime}\right|^{2}\right)+ \\
& +\frac{4 C(\beta) a_{5}}{m_{0}} E_{1}^{3 / 2}+\beta\left|A^{1 / 2} u^{\prime}\right|^{2}-\beta M\left(\left|A^{1 / 2} u\right|\right)|A u|^{2}+\beta a_{3}\left(\frac{\varepsilon_{2}}{2}\left|u^{\prime}\right|^{2}+\frac{1}{2 \varepsilon_{2}}\left|A^{1 / 2} u\right|^{2}\right)
\end{aligned}
$$

Haciendo $\quad \varepsilon_{1}=\frac{k_{0}}{2 C(\beta) a_{3}} ; \quad \varepsilon_{2}=\frac{2 k_{0}}{\beta a_{3}}$, obtenemos

$$
S^{\prime} \leq(\beta-h(2 C(\beta)))\left|A^{1 / 2} u^{\prime}\right|^{2}+\left(\frac{\beta^{2} C_{3}^{3} d_{1}}{4 k_{0} m_{0}}-\beta\right) M\left(\left|A^{1 / 2} u\right|\right)|A u|^{2}+\frac{4 C(\beta) \gamma_{1} C_{5}}{m_{0}} E_{1}^{1 / 2} S
$$

donde

$$
h(2 C(\beta))=2 C(\beta)^{2} \frac{C_{3}^{2}}{k_{0}}-4 k_{0} a_{0} C(\beta)
$$

Debemos hallar $\beta$ tal que:

$$
\begin{aligned}
& h(2 C(\beta))>\beta \\
& \beta<\frac{4 k_{0} m_{0}}{C_{3}^{2} d_{1}}
\end{aligned}
$$


Para $\beta$ suficientemente pequeño se cumplen las condiciones $C(\beta)=\gamma_{0}<\gamma_{1}$ y (30). Para verificar la condición (29), tenemos por hipótesis que:

$$
\lambda=e a=\frac{k_{0}^{2} a_{0} a_{1} d_{1}}{m_{0}}>\frac{1}{2}
$$

Sea $\eta$ número real positivo tal que $\eta>\frac{2 \lambda}{2 \lambda-1}>1$

Consideramos $\beta_{\eta}>0$ tal que $C(\beta)=\frac{a}{2 \eta b}$; entonces $h\left(2 C\left(\beta_{\eta}\right)\right)>0$.

La condición :

$$
\frac{a^{2}}{b} \frac{\eta-1}{\eta^{2}}=h\left(2 C\left(\beta_{\eta}\right)\right)>\beta_{\eta}=C^{-1}\left(\beta_{\eta}\right)
$$

es equivalente (desde que $C$ y $C^{-1}$ son crecientes) con la condición

$$
C\left(\frac{a^{2}}{b} \frac{\eta-1}{\eta^{2}}\right)>\frac{a}{2 \eta b}=C^{-1}\left(\beta_{\eta}\right)
$$

Ahora (33) es consecuencia directa de (31). En efecto;

$$
\begin{aligned}
& \frac{\lambda(\eta-1)}{\eta}>\frac{1}{2} \\
& \frac{d}{b} \frac{a^{3}(\eta-1)^{2}}{\eta^{3}}+\frac{\lambda(\eta-1)}{\eta}>\frac{1}{2} \\
& \frac{a}{\eta b}\left(\frac{d}{b} \frac{a^{3}(\eta-1)^{2}}{\eta^{3}}+\frac{\lambda(\eta-1)}{\eta}\right)>\frac{a}{2 \eta b} \\
& d \frac{a^{4}(\eta-1)^{2}}{b^{2} \eta^{4}}+\frac{e a}{b} \frac{(\eta-1)}{\eta^{2}}=C\left(\frac{a}{b} \frac{\eta-1}{\eta^{2}}\right)>\frac{a}{2 \eta b} .
\end{aligned}
$$

Para $k$ suficientemente grande tenemos que

$$
\gamma_{0}=C\left(\beta_{\eta}\right)=\frac{a}{2 \eta b}<\min \left\{\gamma_{1}, \frac{4 k_{0} m_{0}}{C_{3}^{2} d_{1}}\right\}
$$


Luego

$$
S^{\prime} \leq-p_{k} E_{1}+q E_{1}^{1 / 2} S
$$

donde

$$
\begin{aligned}
& q=\frac{4 C\left(\beta_{\eta}\right) \gamma_{1} C_{5}}{m_{0}} \\
& p=C\left(\beta_{k}\right) \min \left\{\frac{a^{2}}{b} \frac{\eta-1}{\eta^{2}}-\beta_{\eta}, \beta_{\eta}-\beta_{\eta}^{2} \frac{C_{3}^{2} d_{1}}{4 k_{0} m_{0}}\right\}
\end{aligned}
$$

Proposición 3. Si los datos iniciales verifican la condición

$$
\frac{p}{q}>R \geq E_{1}^{1 / 2}(0)
$$

Entonces

$$
\phi(t)=p-q E_{1}^{1 / 2}(t)>0, \quad \forall t \in[0, \infty)
$$

Demostración. Razonando por el absurdo, tenemos que la función $\eta(t)$ es continua y $\eta(0)>0$ por la condición (34). Luego existe $\tau>0$ tal que:

$$
\begin{aligned}
& E_{1}(t)<\left(\frac{p}{q}\right)^{2}=\varepsilon_{0}, \quad \forall t \in[0, \tau) \mathrm{y} \\
& E_{1}(\tau)=\varepsilon_{0}
\end{aligned}
$$

Desde que $S^{\prime}(t) \leq-\phi(t) S(t) \leq 0, \quad \forall t \in[0, \tau)$. Entonces $S(t)-S(0)=\int_{0}^{\tau} S^{\prime}(z) d z \leq 0$, luego $S(\tau) \leq S(0)$.

Por otro lado,

$$
E_{1}(\tau) \leq \gamma_{0}^{-1} S(\tau) \leq \gamma_{0}^{-1} S(0) \leq \gamma_{0}^{-1} \gamma_{1} E_{1}(0) \leq E_{1}(0)<\varepsilon_{0} .
$$

Esto es una contradiccion, luego la proposicion 3 está demostrada.

Acotación para $u_{m}^{\prime \prime}$. En la ecuación aproximada (11), obtenemos

$$
\begin{aligned}
\left|u_{m}^{\prime \prime}\right|^{2} & =-M\left(\left|A^{1 / 2} u_{m}\right|^{2}\right)\left(A u_{m}, u_{m}^{\prime \prime}\right)-\left(B u_{m}^{\prime}, u_{m}^{\prime \prime}\right) \leq \\
& \leq\left(M\left(\left|A^{1 / 2} u_{m}\right|^{2}\right)\left|A u_{m}\right|+\left|B u_{m}^{\prime}\right|\right)\left|u_{m}^{\prime \prime}\right|
\end{aligned}
$$


de donde

$$
\left(u_{m}^{\prime \prime}\right) \text { es acotada en } L^{2}(0, \infty ; H) \text {. }
$$

Con los estimados obtenidos y procediendo de forma estándar para pasar al liímite obtenemos una función $u$ que satisface los requerimientos del teorema 1 .

\section{DECAIMIENTO DE LA ENERGÍA}

En esta seccion demostraremos que el funcional de energía

(39) $E_{1}(t)=\left|A^{1 / 2} u^{\prime}(t)\right|^{2}+M\left(\left|A^{1 / 2} u(t)\right|^{2}\right)|A u(t)|^{2}$

decae exponencialmente cuando $t \rightarrow \infty$.

Sea $0 \leq S \leq T<\infty$. Entonces,

$$
\int_{S}^{T} E_{0}^{\prime}(t) d t=E_{o}(T)-E_{0}(S)=-2 \int_{S}^{T}\left|B^{1 / 2} u^{\prime}(t)\right|^{2} d t \leq 0
$$

Luego,

$$
E(S) \geq E(T)
$$

Por otro lado,

$$
\left(u^{\prime \prime}, u\right)+M\left(\left|A^{1 / 2} u\right|^{2}\right)(A u, u)=\frac{d}{d t}\left(u^{\prime}, u\right)-\left|u^{\prime}\right|^{2}+M\left(\left|A^{1 / 2} u\right|^{2}\right)\left|A^{1 / 2} u\right|^{2}=-\left(B u^{\prime}, u\right) .
$$

De donde,

(41) $\int_{S}^{T}\left|u^{\prime}(t)\right|^{2} d t-\left.\left(u^{\prime}(t), u(t)\right)\right|_{S} ^{T}-\int_{S}^{T} M\left(\left|A^{1 / 2} u\right|^{2}\right)\left|A^{1 / 2} u(t)\right|^{2} d t=\int_{S}^{T}\left(B u^{\prime}(t), u(t)\right) d t$.

También,

$$
E_{0}(t)=\left|u^{\prime}(t)\right|^{2}+\hat{M}\left(\left|A^{1 / 2} u(t)\right|^{2}\right) \leq\left|u^{\prime}(t)\right|^{2}+M\left(\left|A^{1 / 2} u(t)\right|^{2}\right)\left|A^{1 / 2} u(t)\right|^{2},
$$

de donde ,

$$
-\int_{S}^{T} M\left(\left|A^{1 / 2} u(t)\right|^{2}\right)\left|A^{1 / 2} u(t)\right|^{2} d t \leq \int_{S}^{T}\left(\left|u^{\prime}(t)\right|^{2}-E_{0}(t)\right) d t
$$


de (41) y (42) tenemos

$$
0 \leq \int_{S}^{T}\left(\left|u^{\prime}(t)\right|^{2}-E_{0}(t)-\left(B u^{\prime}(t), u(t)\right)\right) d t-\left.\left(u^{\prime}(t), u(t)\right)\right|_{S} ^{T}
$$

de (43)

$$
\int_{S}^{T} E_{0}(t) d t \leq \int_{S}^{T}\left(\left|u^{\prime}(t)\right|^{2}-\left(B u^{\prime}(t), u(t)\right)\right) d t-\left.\left(u^{\prime}(t), u(t)\right)\right|_{S} ^{T}
$$

pero,

$$
\left|u^{\prime}(t)\right|^{2}=\left(u^{\prime}(t), u^{\prime}(t)\right)=\left(B^{-1} u^{\prime}(t), B u^{\prime}(t)\right) \leq k_{0}^{-1}\left|B^{1 / 2} u^{\prime}(t)\right|^{2}=-\frac{k_{0}^{-1}}{2} E_{0}^{\prime}(t)=-C E_{0}^{\prime}(t)
$$

Entonces

$$
\int_{S}^{T}\left|u^{\prime}(t)\right| d t \leq-C \int_{S}^{T} E_{0}^{\prime}(t) d t=-C E_{0}(T)+C E_{0}(S) \leq C E_{0}(S)
$$

también,

$$
\begin{aligned}
\left|\left(B u^{\prime}(t), u(t)\right)\right| & \leq k_{1}\left|u^{\prime}(t)\right||u(t)| \leq k_{1} a_{2}\left|u^{\prime}(t)\right|\left|A^{1 / 2} u(t)\right| \leq \\
& \leq \frac{k_{1} a_{2}}{2}\left\{\left|u^{\prime}(t)\right|^{2}+\left|A^{1 / 2} u(t)\right|^{2}\right\} \leq C E_{0}(t)
\end{aligned}
$$

de donde

$$
\int_{S}^{T}\left(B u^{\prime}(t), u(t)\right) d t \leq C_{8} \int_{S}^{T} E_{0}(t) d t
$$

Asimismo, tenemos el siguiente estimado

$$
\text { (47) } \begin{aligned}
-\left.\left(u^{\prime}(t), u(t)\right)\right|_{S} ^{T} & =\left(u^{\prime}(S), u(S)\right)-\left(u^{\prime}(T), u(T)\right) \leq\left|u^{\prime}(S)\right||u(S)|+\left|u^{\prime}(T)\right||u(T)| \leq \\
& \leq C E_{0}(S)+C \quad E_{0}(T) \leq 2 C E_{0}(S)
\end{aligned}
$$

Por lo tanto de (44), (45), (46) y (47) obtenemos finalmente

$$
\int_{S}^{T} E_{0}(t) d t \leq C E_{0}(S)
$$

Con lo cual se demuestra el decaimiento de la energía en forma exponencial. 


\section{REFERENCIAS BIBLIOGRAFICAS}

[1] AROSIO A. \& SPAGNOLO S. "Global solutions of the Cauchy problem for a non- linear Hyperbolic Equation ". Universita di Pisa . Departamento de Matemática. Roma (1982).

[2] A'ASSILA M. "On a Quasilinear Wave Equation with Strong Damping". Funkcialaj Ekvaioj 41 (1998).

[3] COUSIN, A., FROTA, C., LARKIN, N. \& MEDEIROS, L. A. "On the abstrac model of Kirchhoff-Carrier Equation" Comm. In Appl. Analysis 3 (1997).

[4] EBIHARA, Y. "On the existence of local smooth solutions for same degenerate quasilinear hyperbolic equations". Anais Acad. Bras. Ciencias. Vol. 57 (1985).

[5] EBIHARA, Y., MEDEIROS, L. \& MILLA, A. "Local solutions for a nonlinear degenerate hyperbolic equations". Nonlinear Analysis. Vol. 10. (1986).

[6] IZAGUIRRE, R. \& PEREIRA, D. "Solução global para uma classe de equações hiperbólicas degeneradas". Proceeding of $9^{0}$ Congreso Brasileiro de Matemática Aplicada e Computacional. Brasilia (1986).

[6'] IZAGUIRRE, R. \& CABANILLAS, E.. "Solución Global para una Clase de Ecuaciones Abstractas Degeneradas Asociadas a la Ecuación No Lineal de la Viga" . Por aparecer.

[7] IZAGUIRRE, R. \& VELIZ, V. "Existencia y Unicidad de Solución Local para una Clase de Ecuaciones Abstractas Degeneradas No-Lineales tipo Kirchhoff-Carrier" I Seminario Internacional de Ecuaciones Diferenciales Parciales y Aplicaciones. Universidad Ricardo Palma Perú (1999).

[8] IZAGUIRRE, R. \& VELIZ, V. "Solución Local para una clase de ecuaciones tipo Kirchoff". Actas del $42^{\circ}$ Seminario Brasilero de analisis (1997).

[9] LIMACO, J. \& FERREL, L. "Existência de soluções para a equação da corda clástica com amortecimento". Atas do $37^{\circ}$ Seminario Brasileiro de Analise.

[10] LIONS, J. L. "Quelques Methodes de Resolution des Probleme aux limites nonlinear". Dunod. Paris. (1969).

[11] MATOS, M. "Estudo de um modelo abstrato para a equação da viga, via 
integral Hilbertiana". Atas do $29^{\circ}$ Seminario Brasileiro de Analise (1989).

[12] MEDEIROS, L.A \& MILLA, M.M. "Remarks on a nonlinear model vibrations of string with damping". $30^{\circ}$ Seminario Brasilero de Analise. L.N.C.C. R.J. (1989).

[13] MUÑOZ, J. "Remark on the existence and Decay of the Nonlinear Beam Equation". Internat. J. Math. Vol 17 , N0 2 (1994).

[14] PEREIRA, D. C. "Existence, Uniqueness and Asymptotic Behavior for Solutions of the nonlinear Beam equation". Nonlinear Analysis, Theory, Methods and Applications, Vol 14, N0 8, 1990.

[15] PERLA, G. "On classical solutions of quasilinear hyperbolic equations". Nonlinear Analysis. Vol. 3. (1979).

[16] RIVERA, P. “On local strong solutions of a nonlinear partial differential equation". Appl. Analysis. Vol. 10. (1980).

[17] WOINOWSKY, S. Y KRIEGER. "The effect of axial force on the vibration of hinged bar" J. Appl. Math. 17 (1950). 